\title{
High Resolution CT following Primary Spontaneous Pneumothorax in Adolescents: Useful tool or wasted radiation? Dr. Sarah Stanko, MD University of Arizona, Dr. Colette Oesterle, MD University of Arizona, Dr. Merlin Lowe, MD University of Arizona Keywords: primary spontaneous pneumothorax, pneumothorax, bleb, adolescent, pediatrics, chest tubes Correspondence: Dr. Merlin Lowe Dept. of Pediatrics 1501 N. Campbell Ave. Tucson, AZ 85724 520-626-6614 520-626-2883 lowe@peds.arizona.edu
}

\author{
Sarah Stanko ${ }^{1}$, Merlin Lowe ${ }^{2}$, and Colette Oesterle ${ }^{1}$ \\ ${ }^{1}$ University of Arizona Medical Center - Diamond Children's \\ ${ }^{2}$ The University of Arizona College of Medicine Tucson
}

January 21, 2021

\begin{abstract}
Background The current trend in management of first-time primary spontaneous pneumothorax (PSP) in children is to obtain a high resolution chest computerized tomography (HRCT) scan to look for bleb/bullae disease or other structural lung disease. We aimed to evaluate the significance of HRCT findings in relation to initial management strategies, and we hypothesized that these findings do not guide management. Methods We evaluated patients with first time PSP in a single-institution, retrospective, longitudinal study. The primary endpoint was the percentage of patients who underwent surgical intervention after HRCT. Results We identified ten children from 10-17 years old from January 2013 to November 2019 who met criteria for the study. Seven out of ten patients $(70 \%)$ had HRCT after first-time PSP during the same hospital stay. Blebs/bullae were discovered in five out of seven (71\%) of those patients. Two of those five patients had subsequent surgical intervention (40\%). $28 \%$ of those who had a HRCT had surgery at that point. Of those treated conservatively with blebs/bullae identified on HRCT, $66 \%$ had recurrence of PSP and all patients subsequently underwent VATS with blebectomy and pleurodesis. Among the patients without initial HRCT, there were no recurrent cases of PSP noted. Conclusions There is value in obtaining HRCT after the first time PSP, as these results can be used to guide management strategies. Further studies in pediatric PSP are needed to validate the sensitivity of HRCT in bleb detection, the predictive value of bleb disease and recurrence, and the benefits and risks of early surgical intervention
\end{abstract}

\section{Hosted file}

High Resolution CT following Primary Spontaneous Pneumothorax in Adolescents.pdf available at https://authorea.com/users/390963/articles/505170-high-resolution-ct-followingprimary-spontaneous-pneumothorax-in-adolescents-useful-tool-or-wasted-radiation-drsarah-stanko-md-university-of-arizona-dr-colette-oesterle-md-university-of-arizonadr-merlin-lowe-md-university-of-arizona-keywords-primary-spontaneous-pneumothoraxpneumothorax-bleb-adolescent-pediatrics-chest-tubes-correspondence-dr-merlin-lowe-dept- 
of-pediatrics-1501-n-campbell-ave-tucson-az-85724-520-626-6614-520-626-2883-lowe-pedsarizona-edu

\section{Hosted file}

Spontaneous PNX Tables.pdf available at https://authorea.com/users/390963/articles/ 505170-high-resolution-ct-following-primary-spontaneous-pneumothorax-in-adolescentsuseful-tool-or-wasted-radiation-dr-sarah-stanko-md-university-of-arizona-dr-coletteoesterle-md-university-of-arizona-dr-merlin-lowe-md-university-of-arizona-keywordsprimary-spontaneous-pneumothorax-pneumothorax-bleb-adolescent-pediatrics-chest-tubescorrespondence-dr-merlin-lowe-dept-of-pediatrics-1501-n-campbell-ave-tucson-az-85724520-626-6614-520-626-2883-lowe-peds-arizona-edu 